\title{
Two-Parameter Bifurcation Analysis of the Buck Converter*
}

\author{
Alessandro Colombo ${ }^{\dagger}$, Paola Lamiani ${ }^{\dagger}$, Luis Benadero $\ddagger$, and Mario di Bernardo ${ }^{\S}$
}

\begin{abstract}
This paper is concerned with the analysis of two-parameter bifurcation phenomena in the buck power converter. It is shown that the complex dynamics of the converter can be unfolded by considering higher codimension bifurcation points in two-parameter space. Specifically, standard smooth bifurcations are shown to merge with discontinuity-induced bifurcation (DIB) curves, giving rise to intricate bifurcation scenarios. The analytical results are compared with those obtained numerically, showing excellent agreement between the analytical predictions and the numerical observations. The existence of these two-parameter bifurcation phenomena involving DIBs and smooth bifurcations, predicted in [P. Kowalczyk et al., Internat. J. Bifur. Chaos Appl. Sci. Engrg., 16 (2006), pp. 601-629; A. Colombo and F. Dercole, SIAM J. Appl. Dyn. Syst., submitted], is confirmed in this important class of systems.
\end{abstract}

Key words. bifurcation, discontinuity, nonsmooth, buck, power converter, codimension-two

AMS subject classifications. $37 \mathrm{~N} 35,34 \mathrm{C} 23,37 \mathrm{G} 15$

DOI. $10.1137 / 080741434$

1. Introduction. Step-down switching converters (buck converters) are popular circuits in modern electronics. Even though they are larger and noisier than linear regulators, they offer indisputable advantages in terms of energy efficiency, a feature that is becoming more and more important with the enormous popularity of electronic handheld devices, which require many different voltage levels to be supplied by the same battery with minimal waste of energy. In its simplest form, which is the one we consider in this paper, the buck converter consists of a diode, an inductor, a capacitor, and a switch that is suitably controlled to generate an output voltage with the desired average value (see, for example, [23] for details). In its nominal operating conditions the circuit is assumed to work on a limit cycle, but even limiting the analysis to the simplest types of periodic orbits - which are the most common in applicationsthe number of qualitatively different orbits found in the system can be very large $[20,2,3,1]$. Rather than recurring to averaging methods, which are the standard tool for the analysis of this kind of circuit, in this paper we provide a detailed analysis of the complete model and of its bifurcations, highlighting regions in parameter space where multiple asymptotic behaviors, or even aperiodic dynamics, are possible.

We model the circuit as a piecewise smooth system (see [19] for a classical introduction to this topic), that is to say, a set of ordinary differential equations with a piecewise smooth vector

\footnotetext{
* Received by the editors November 20, 2008; accepted for publication (in revised form) by W.-J. Beyn August 11, 2009; published electronically November 5, 2009.

http://www.siam.org/journals/siads/8-4/74143.html

${ }^{\dagger}$ DEI, Politecnico di Milano, Milan, Italy (alessandro.colombo@polimi.it, smile_paolam@hotmail.it).

${ }^{\ddagger}$ Department of Applied Physics, Universitat Politécnica de Catalunya (UPC), Barcelona, Spain (luis@fa.upc.edu).

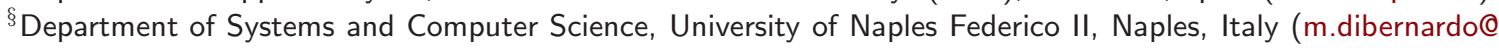
bristol.ac.uk).
} 


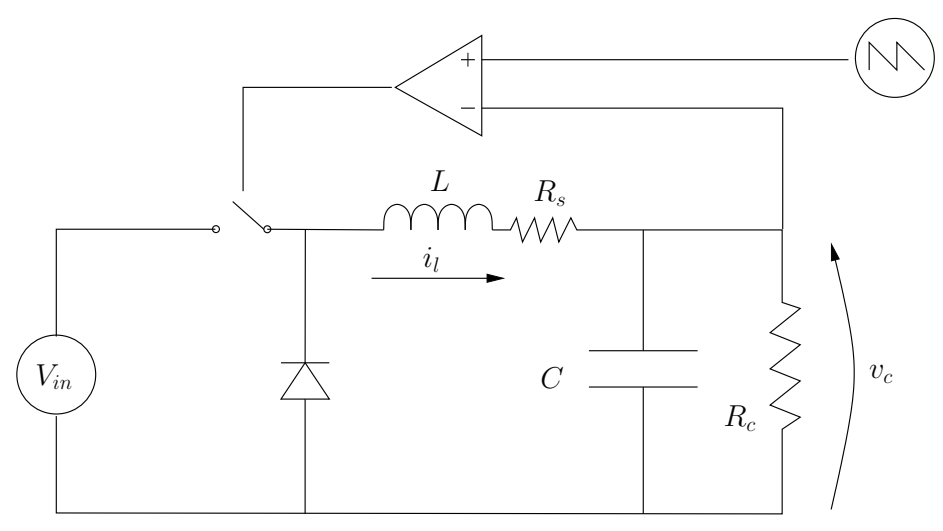

Figure 1. Schematic diagram of the buck DC-DC converter with voltage mode control

field. Bifurcations of piecewise smooth systems are a major subject of research in the recent literature, the most comprehensive survey being [11], but the numerical and analytical tools that can be used to study these bifurcations are still very limited. For this reason, we devote particular attention to the analysis of these bifurcations that, as appears from the results presented herein, represent a key ingredient to the understanding of this model's dynamics. We focus our attention on the occurrence of so-called discontinuity induced bifurcations (or DIBs), which are unique to discontinuous systems.

It is worth mentioning here that the circuit studied in this paper serves as an excellent representative example to highlight the presence in piecewise smooth dynamical systems of a novel class of two-parameter DIB, whose occurrence was conjectured in [21, 8]. According to the classification presented therein, we show that the buck converter under investigation exhibits two fundamental types of two-parameter bifurcation phenomena: those where a smooth bifurcation curve (e.g., fold or flip) merges with curves of DIBs, and those characterized by the simultaneous occurrence of more than one DIB. In both cases, we show how the associated codimension-two points in parameter space are fundamental in organizing the rest of the circuit's dynamics.

In contrast to previous papers reporting two-parameter bifurcation diagrams for the buck (e.g., [6, 3, 22]), our analysis is based on the numerical continuation of the smooth and nonsmooth bifurcations of the model, carried out with methods borrowed from the theory of standard bifurcations, and suitably adapted to the analysis of DIBs. The mathematical details are reported in this paper.

2. The model. Among the many possible implementations of the buck converter, here we focus on the pulse-width-modulated buck DC-DC converter with voltage mode control, whose circuit is schematized in Figure 1. The power stage consists of seven basic elements: the input voltage $V_{i n}$, a controlled switch (usually a MOSFET), a diode, the inductor $L$ that stores energy and transfers it from the input to the output, the filter capacitor $C$ that flattens the voltage output, and two resistors. Resistor $R_{s}$ models the inductor losses, while $R_{c}$ represents the load, which is assumed to be purely resistive. Additionally, a comparator (the triangle in Figure 1) opens or closes the switch when the voltage $v_{c}$ becomes higher or lower than a given, 
time-dependent, threshold signal (the circled sawtooth in Figure 1). The converter operates by opening and closing the switch repetitively, thus connecting and disconnecting the input voltage from the circuit. When the switch is open, the current passing through the inductor comes from the diode; on the other hand, when the switch is closed, the current is entirely provided by the input voltage, and the diode acts as an open switch.

Let $v_{c}$ be the capacitor (or output) voltage, and $i_{l}$ the inductor current. To gain some intuition on this circuit's behavior, we can assume at first that $R_{s}=0$ and that $v_{c}$ remains constant at some fixed value to be determined. Then, $i_{l}$ changes linearly with time, increasing when the switch is closed and decreasing when it is open. Assuming that $i_{l}$ is periodic, the ratio of $v_{c}$ to $V_{i n}$ is equal to the duty ratio of the switch, that is, the percentage of time during which the switch is closed. This ratio can in turn be regulated by changing the characteristics (frequency, mean value, and amplitude) of the threshold signal that inputs the comparator in Figure 1. Additionally, the power level that the converter must provide may influence the choice of the threshold signal and of the other components. Typically in high-power applications, where the current ripple must be small to minimize losses and electromagnetic interference, the components and the threshold signal are chosen so that the current is always strictly positive. In this case, the circuit is said to work in continuous conduction mode (CCM). On the other hand, for low-power applications, where opening and closing of the switch causes a significant amount of the overall losses, slower switching frequencies are used, letting the current go to zero. In this case the circuit is said to work in discontinuous conduction mode (DCM). The limit between CCM and DCM, shown in the bifurcation diagrams in section 4.1, is reached when $i_{l}$ falls to zero and $v_{c}$ crosses the threshold at the same time.

The circuit in Figure 1 can be modeled as a piecewise smooth system of the form

$$
\dot{x}=f^{(j)}(x)
$$

with three vector fields: $f^{(1)}(x)$, describing the dynamics when the switch is on; $f^{(0)}(x)$, when the switch is off and the circuit operates in CCM; and $f^{(s)}(x)$, when the switch is off and the circuit operates in DCM. In the last case we have named the vector field $f^{(s)}(x)$, as for sliding, because when the current goes to zero the state of the system is bound to slide on the surface $i_{l}=0$. The state vector $x$ is composed of the two electrical variables $v_{c}$ and $i_{l}$, plus the phase of the threshold signal, which is periodic. Hence the state space is isomorphic to $\mathbb{R}^{2} \times S$.

In the following, in order to reduce the number of parameters, we write the system in nondimensional variables following [3] (details are reported in the appendix). The nondimensional equivalent of voltage, current, and phase are named, respectively, $v, i$, and $\theta$. The three vector fields are affine and equal to

$$
f^{(j)}(x)=A_{j} x+B_{j}
$$

with

$$
\begin{array}{cc}
x=\left[\begin{array}{c}
v \\
i \\
\theta
\end{array}\right], \quad A_{1}=A_{0}=\left[\begin{array}{ccc}
-\frac{2 \pi}{Q} & 2 \pi & 0 \\
-2 \pi & -\frac{2 \pi}{Q_{S}} & 0 \\
0 & 0 & 0
\end{array}\right], \quad A_{s}=\left[\begin{array}{ccc}
-\frac{2 \pi}{Q} & 0 & 0 \\
0 & 0 & 0 \\
0 & 0 & 0
\end{array}\right], \\
B_{1}=\left[\begin{array}{c}
0 \\
2 \pi \\
1
\end{array}\right], \quad B_{0}=B_{s}=\left[\begin{array}{l}
0 \\
0 \\
1
\end{array}\right] .
\end{array}
$$

Copyright $\odot$ by SIAM. Unauthorized reproduction of this article is prohibited. 


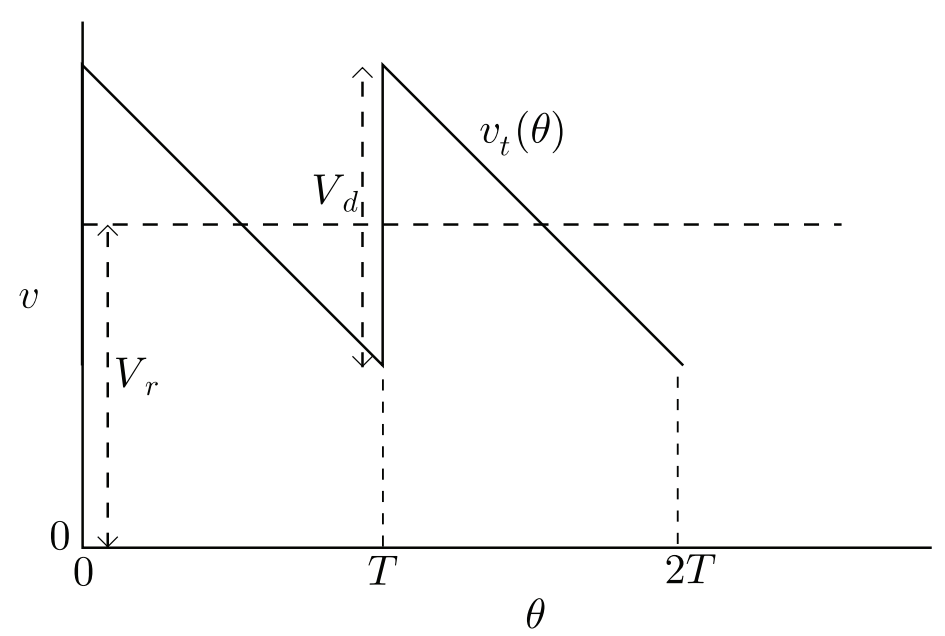

Figure 2. The threshold signal $v_{t}(\theta)$ is assumed to be a sawtooth of period T, with "peak-to-peak" (troughto-peak) amplitude $V_{d}$ and mean value $V_{r}$.

The system switches between the vector fields $f^{(0)}(x)$ and $f^{(1)}(x)$ whenever $v$ becomes higher or lower than the threshold, here called $v_{t}(\theta)$, which is assumed to be a negative sawtooth function with mean value $V_{r}$, peak-to-peak amplitude $V_{d}$, and period $T$ (see Figure 2):

$$
v_{t}(\theta)=V_{r}+V_{d}\left(\frac{1}{2}-\left(\frac{\theta}{T} \bmod 1\right)\right) .
$$

It switches, instead, from $f^{(0)}(x)$ to $f^{(s)}(x)$ when $i$ goes to zero. These "switching surfaces" belong to the zero sets of the following functions:

$$
\begin{array}{lll}
H_{d}(x): x \rightarrow v-V_{d}\left(\frac{1}{2}-\frac{\theta}{T}\right) & \bmod V_{r} & \text { (slanted segment of the threshold) }, \\
H_{v}(x): x \rightarrow \theta \quad \bmod T & \text { (instantaneous reset of the threshold) }, \\
H_{s}(x): x \rightarrow i & \text { (zero current across the diode) },
\end{array}
$$

which are used throughout the analysis. Notice that the threshold $v_{t}(\theta)$ is contained in the union of two separate and smooth switching functions, $H_{d}$ and $H_{v}$, accounting, respectively, for the slanted and vertical parts of the threshold signal. A point $x=(v, i, \theta)$ lies on $v_{t}(\theta)$ when $H_{d}(x)$ or $H_{v}(x)$ is equal to 0 , and $V_{r}-V_{d} / 2 \leq v \leq V_{r}+V_{d} / 2$.

3. Bifurcations: Mathematical details. In this section we explain the mathematical details needed to derive the bifurcation diagrams of our model. First, notice that the three vector fields $f^{(0)}(x), f^{(1)}(x)$, and $f^{(s)}(x)$ admit "stationary" orbits (that is, orbits along which the electrical variables $v$ and $i$ remain constant), which we call $X^{0}$ and $X^{1}$, where $X^{0}$ is an orbit of both $f^{(0)}(x)$ and $f^{(s)}(x)$, and $X^{1}$ is an orbit of $f^{(1)}(x)$. The corresponding values of $i$ and $v$ are, respectively,

$$
(v, i)=(0,0)
$$

Copyright $\odot$ by SIAM. Unauthorized reproduction of this article is prohibited. 

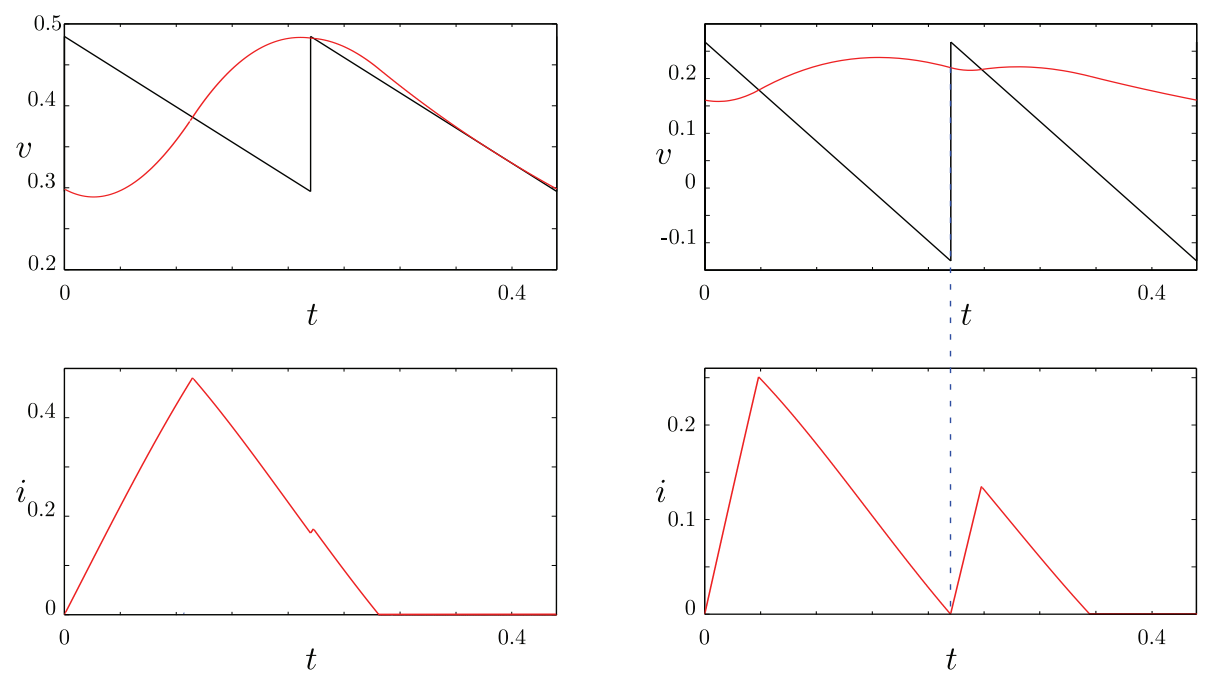

Figure 3. Voltage and current values of period T cycles undergoing a grazing bifurcation (left panels) and a boundary-intersection crossing bifurcation (right panels). In the boundary-intersection crossing, the blue line highlights the simultaneous crossing of the threshold and of the surface $i=0$.

for $X^{0}$ and

$$
(v, i)=\left(\frac{Q Q_{s}}{1+Q Q_{s}}, \frac{Q_{s}}{1+Q Q_{s}}\right)
$$

for $X^{1}$. Notice that $f^{(s)}(x)$ admits an infinite number of stationary orbits, but only $X^{0}$ satisfies the constraint $i=0$. If either $X^{0}$ or $X^{1}$ is entirely contained in the region of definition of the respective vector field, then it is also a solution of system (1). These solutions are not interesting in applications, and under suitable conditions (detailed in the next section) both are not admissible. In this case, the system must settle either on a limit cycle of period $n T$ for some natural $n$, or on more complex attractors. The regions of existence of these attractors are separated by an intricate set of bifurcations, including both smooth bifurcations, like flips and tangent bifurcations of cycles, and DIBs (see [11]), that involve the nontrivial interaction of attractors with the discontinuity boundaries. Smooth bifurcations can be analyzed and continued using standard methods (see, for example, $[13,14,4]$ ), while here we report the conditions that define the two types of DIBs present in the model. In all cases, we suppose that a periodic orbit of period $n T$ exists, thus satisfying the equations

$$
\begin{aligned}
\dot{x}(t)-f^{(j)}(x(t), p) & =0, \\
x(0)-x(n T) & =0 .
\end{aligned}
$$

3.1. Boundary-intersection crossing. This bifurcation takes place when a periodic orbit simultaneously crosses two intersecting discontinuity boundaries, as in the examples in Figure 3 (right panels). In particular, in our model this can happen when an orbit touches a corner of the threshold signal, or when it crosses the zero-current surface and the threshold at the same time. This last case in particular marks the transition between CCM and DCM. In the 
first case, assuming that the cycle crosses the threshold at $t=0$, a bifurcating cycle of period $n T$ satisfies the following conditions:

$$
\begin{aligned}
& H_{v}(x(0), p)=0, \\
& H_{d}(x(0), p)=0,
\end{aligned}
$$

with the additional constraint

$$
V_{r}-\frac{V_{d}}{2} \leq v(0) \leq V_{r}+\frac{V_{d}}{2}
$$

where (5) and (6) say that the cycle must cross both $H_{v}=0$ and $H_{d}=0$ at $t=0$. In the second case, one just has to substitute $H_{s}(x(0), p)$ for $H_{d}(x(0), p)$ in $(6)$.

Different instances of these bifurcations are studied in $[11,7]$, where it is proved that the Poincaré map of the bifurcating cycle can be linearized and is continuous (but has in general a discontinuous derivative). Depending on the eigenvalues of the linearized Poincaré map, as explained in section 5, we know whether the cycle collides at the bifurcation point with another cycle or whether it continues to exist through the bifurcation, and whether a cycle of double period is born at the bifurcation point. The stability of all the attractors can be deduced from the eigenvalues of the linearized Poincaré map.

3.2. Grazing. In this case, a periodic orbit touches tangentially a discontinuity boundary. In the bifurcation diagrams we show hereafter, this happens only for an orbit in $f^{(0)}(x)$ that is tangent to the slanted part of the threshold (which is the zero set of $H_{d}$ ), as shown in Figure 3 (left panels). Therefore we report the conditions only for this particular case. Again, assuming that the grazing occurs at $t=0$, these conditions become

$$
\begin{aligned}
H_{d}(x(0), p) & =0, \\
\left\langle f^{(0)}(x(0), p), \frac{d}{d x} H_{d}(x(0), p)\right\rangle & =0,
\end{aligned}
$$

with the constraint

$$
V_{r}-\frac{V_{d}}{2} \leq v(0) \leq V_{r}+\frac{V_{d}}{2}
$$

where $\langle\cdot, \cdot\rangle$ denotes the scalar product and the two equations express the condition of tangency between the trajectory and the surface of discontinuity. In this case, [10] showed that, as the system has discontinuous vector fields across the switching manifold and no sliding, the Poincaré map has a square root singularity at the bifurcation point. As a consequence the bifurcation is often associated with the birth of complex attractors.

The easiest way to detect and continue both smooth bifurcations and the DIBs listed above is to reduce the system to a discrete-time map of the form

$$
x(k+1)=\phi(x(k), T, p),
$$

where $\phi(x(k), T, p)$ is the flow of $x(k)$ after a time $T$, depending on the parameter vector $p$. If $H_{v}(x(0))=0$, this is the $T$-periodic stroboscopic map of the model evaluated at the reset of the threshold. Since all vector fields are affine, this map is given implicitly by a system of 
$2 N-1$ transcendental equations, where $N$ is the number of vector fields visited by the orbit during a period $T$ of the threshold (details can be found in [12]). In this case the periodicity conditions stated above become

$$
\begin{array}{r}
x(k+n)-\phi(x(k), n T, p)=0, \\
x(k+n)-x(k)=0,
\end{array}
$$

while the defining conditions of the bifurcations described above are

$$
\begin{array}{r}
H_{d}(x(k), p)=0 \quad \text { or } \quad H_{s}(x(k), p)=0, \\
V_{r}-\frac{V_{d}}{2} \leq v(k) \leq V_{r}+\frac{V_{d}}{2}
\end{array}
$$

for the boundary-intersection crossing, and

$$
\begin{gathered}
H_{d}\left(\phi\left(x(k), t^{*}, p\right), p\right)=0, \\
\left\langle f^{(0)}\left(\phi\left(x(k), t^{*}, p\right), p\right), \frac{d}{d x} H_{d}\left(\phi\left(x(k), t^{*}, p\right), p\right)\right\rangle=0, \\
V_{r}-\frac{V_{d}}{2} \leq v^{*} \leq V_{r}+\frac{V_{d}}{2}, \\
0<t^{*}<n T,
\end{gathered}
$$

for the grazing. Here, $v^{*}$ is the voltage value of $\phi\left(x(k), t^{*}, p\right)$.

\section{Bifurcation analysis.}

4.1. Numerical continuation. Since the buck converter is commonly assumed to operate on limit cycles of period close to that of the threshold signal, we focus our analysis on cycles of period $T$ and $2 T$. Note that most existing software cannot be used for continuation of solutions of a piecewise smooth set of differential equations, with the exception of TC-HAT [25], which appeared during the writing of this paper, and SlideCont [9], which cannot handle all bifurcations found in this model. Hence, using the conditions given above, ad hoc numerical continuation routines were written in Maple to trace the bifurcation curves in two parameters. We present two-dimensional bifurcation diagrams in the $\left(V_{r}, V_{d}\right)$ plane $\left(-0.4 \leq V_{r} \leq 1.4\right.$, $\left.0 \leq V_{d} \leq 0.7\right)$ using the following parameter set: $Q=2.5, Q_{s}=15$, and $T=0.22$. These parameters, though physically realistic, are not used (to our knowledge) in any particular real circuit. They were chosen nonetheless because they allow us to observe a rich set of dynamics. For a clearer exposition, we represent in Figures 4, 6, and 7 only the bifurcations of stable invariant sets, omitting all details regarding the unstable invariant sets that are nevertheless involved. Only in Figure 8 is a complete picture of the stable and unstable invariant sets involved in each bifurcation given for the central part of the bifurcation diagram.

First, we shall identify the region in the $\left(V_{r}, V_{d}\right)$ plane where the two stationary solutions $X^{0}$ and $X^{1}$ (see (3) and (4)) do not exist, so that the electrical variables must oscillate. This happens when the voltage value of $X^{0}$ is lower than the maximum value of the threshold, and the voltage value of $X^{1}$ is higher than the minimum value of the threshold. Therefore, the system has no stationary solutions if the following conditions are both verified (see Figure 4):

$$
V_{r}+\frac{V_{d}}{2}>0, \quad V_{r}-\frac{V_{d}}{2}<\frac{Q Q_{s}}{1+Q Q_{s}} .
$$

Copyright $\odot$ by SIAM. Unauthorized reproduction of this article is prohibited. 


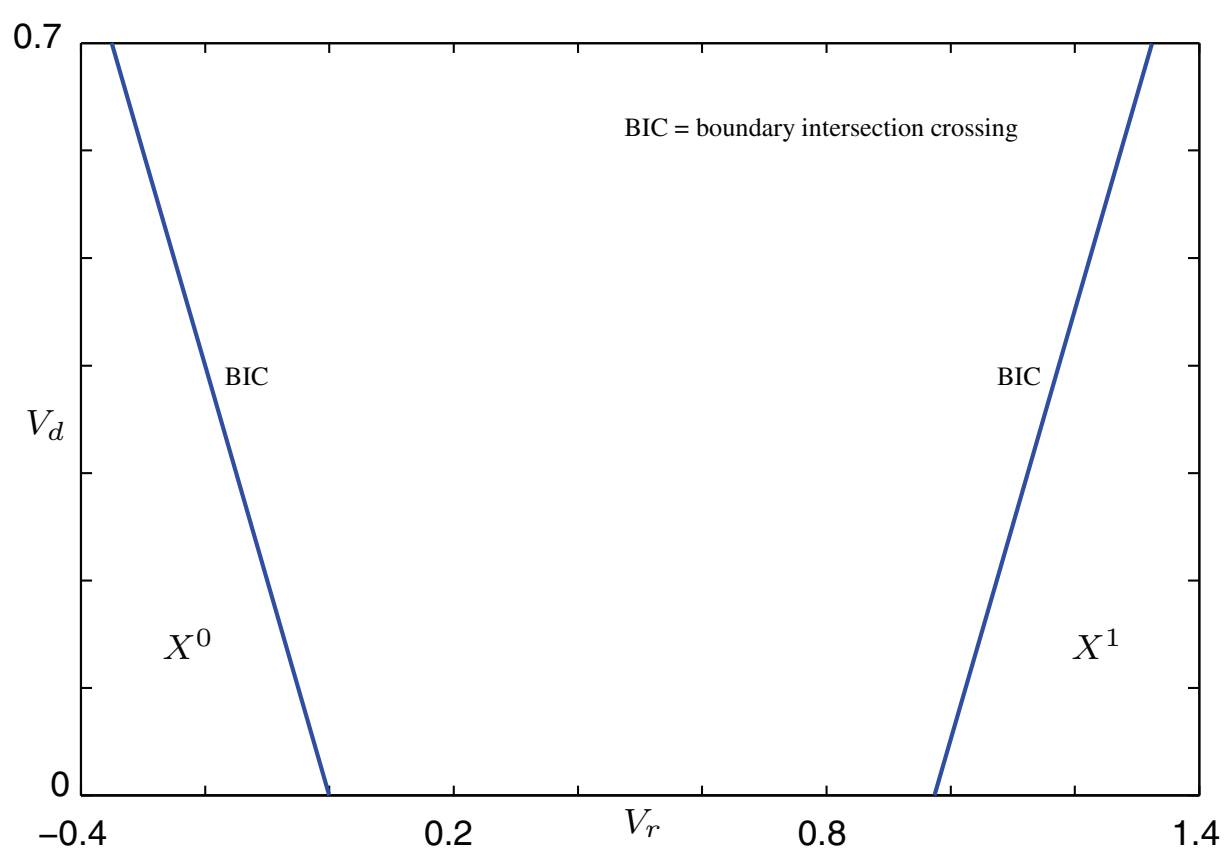

Figure 4. The blue lines represent bifurcations of the analyzed attractors. In this case, the regions of existence of two stationary solutions $X^{0}$ and $X^{1}$ are delimited by two boundary-intersection crossing curves.
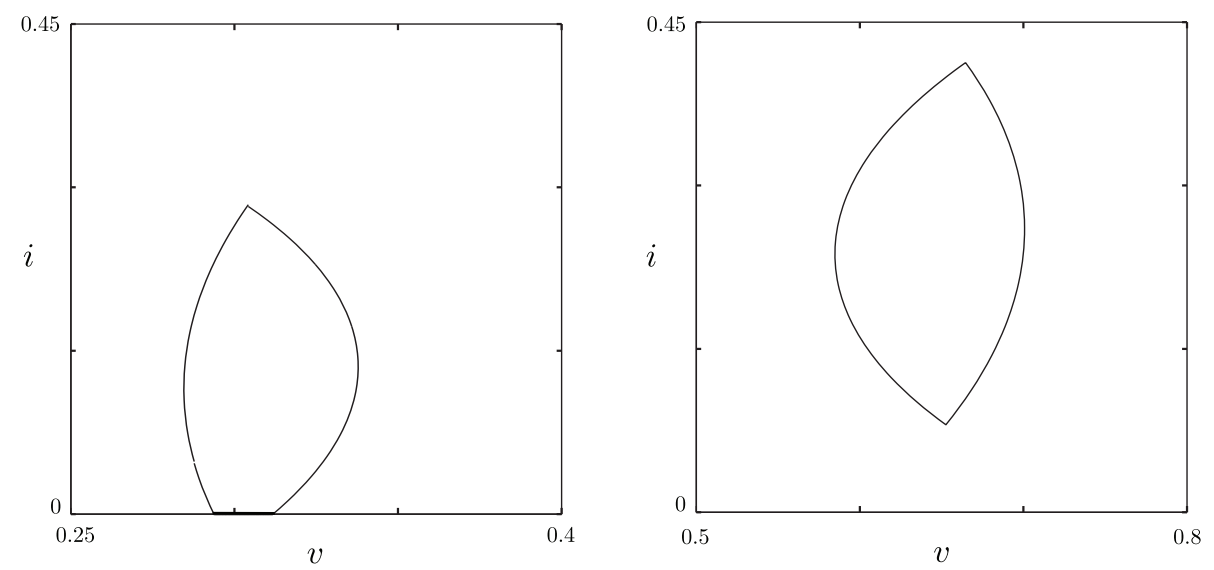

Figure 5. Two cycles obtained for $Q=2.5, Q_{s}=15$, and $T=0.22$ and different values of $V_{r}$ and $V_{d}$. The one in the left panel slides along the surface $i=0$ for a fraction of its period, and therefore the converter works in DCM, while the one on the right has strictly positive current, so that the converter works in CCM.

On the border of this region, the only admissible stationary solution (either $X^{0}$ or $X^{1}$ ) disappears through a boundary-intersection crossing with the corners of the threshold signal, while inside the region two qualitatively different cycles of period $T$ may exist: one that slides on $H_{s}(x)=0$ for a fraction of its period, and one that does not, as shown in Figure 5. The transition from the CCM cycle to the DCM one is given by the boundary-intersection crossing curve in the center of Figure 6. In this figure and those that follow, we describe with a 


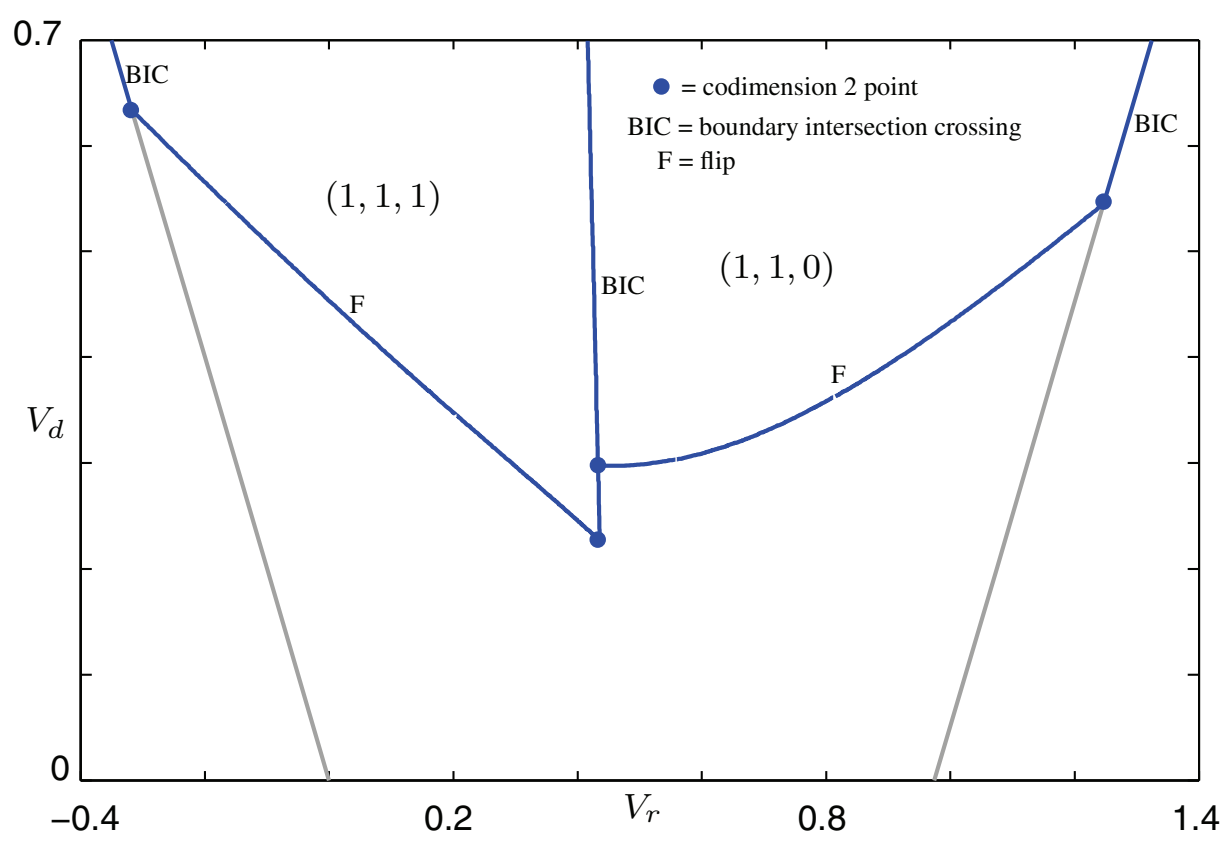

Figure 6. Two stable cycles of period $T$ exist in the upper part of the bifurcation diagram and are separated by a curve of boundary-intersection crossing.

string $(n, m, p)$ a cycle that has period $n T$, that intersects the threshold $2 m$ times during one period, and that has $p$ distinct segments on the surface $i=0$. Thus, the cycles in Figure 5 have strings $(1,1,0)$ and $(1,1,1)$, respectively. As we see in Figure 6 , the region of existence and stability of these $T$-periodic orbits is bounded from below by two flip curves, and on the side by two boundary-intersection crossings, which coincide with the limit of existence of the stationary solutions. As we mentioned in the previous section, these two cycles are the most commonly used in applications, and, in particular, (1,1,0)-type cycles are chosen in highpower applications where the current ripple must be minimized, whereas $(1,1,1)$-type cycles are used in low-power applications, where working in DCM allows the use of low-frequency threshold signals and hence reduces the losses caused by switching.

Along the boundary-intersection crossing curve in the center of Figure 6 we encounter three codimension-two points (see Figure 7): two grazing-flip and one grazing-fold. Following the curves that emanate from these points, and their subsequent branchings, we can isolate the regions of existence of four qualitatively different attractors of period $2 T$. Notice that the cycles $(2,1,1)$ have period $2 T$ and only two switches per period, and so they have half the switching frequency of any cycle of period $T$ (which must switch twice per period). These could therefore be of interest in cases where low switching frequencies are preferable. In the center of the figure, some of these cycles coexist with one of period $T$. A schematic blowup of the center of the bifurcation diagram is given in Figure 8, where the codimension-two points mentioned above are labeled $P_{1}, P_{2}$, and $P_{3}$, and all the invariant sets (stable and unstable) involved in each bifurcation are detailed. Moving further down in the diagram, an intricate set of smooth and nonsmooth bifurcations separates attractors of longer period and 


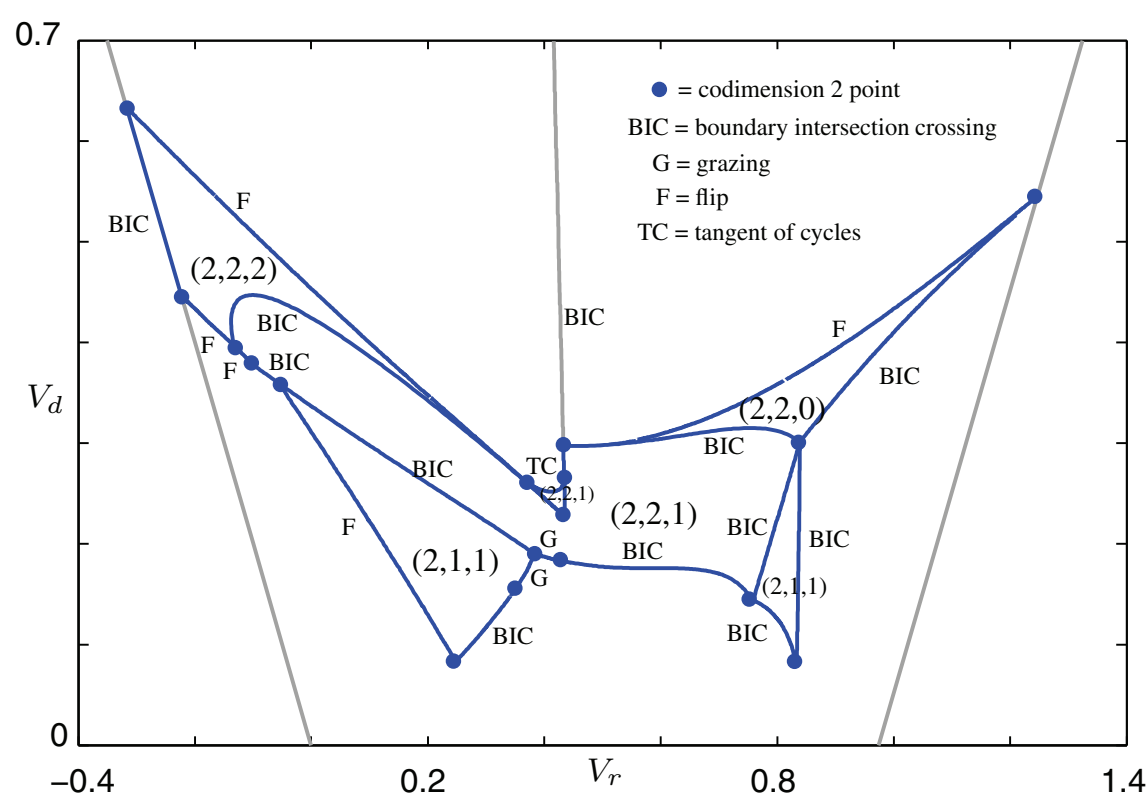

Figure 7. Bifurcation curves of the cycles of period $2 T$.

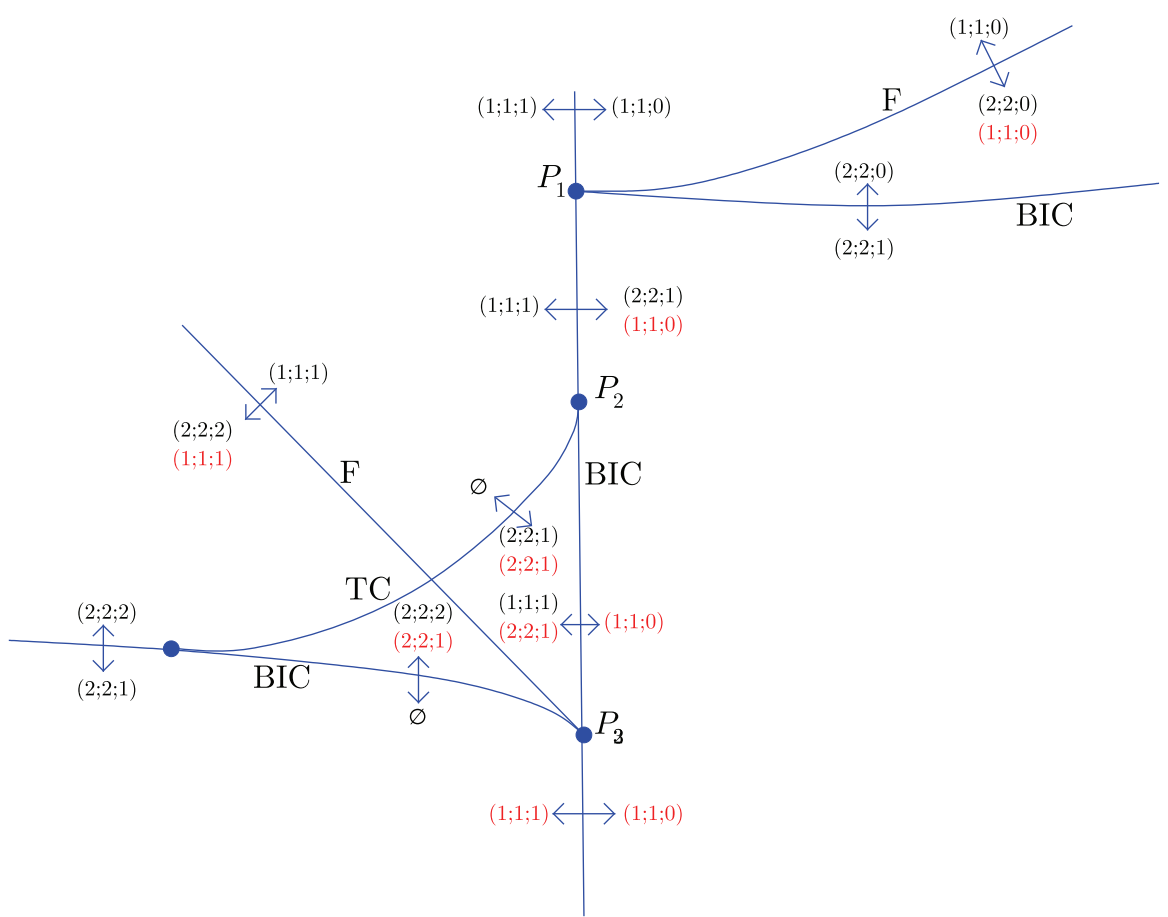

Figure 8. Schematic diagram of the bifurcation curves in the center of Figure 7. For each curve a double arrow points out the stable (in black) and unstable (in red) invariant sets involved on the two sides of the bifurcation. The curves have been stretched to make the diagram more readable.

Copyright (c) by SIAM. Unauthorized reproduction of this article is prohibited. 


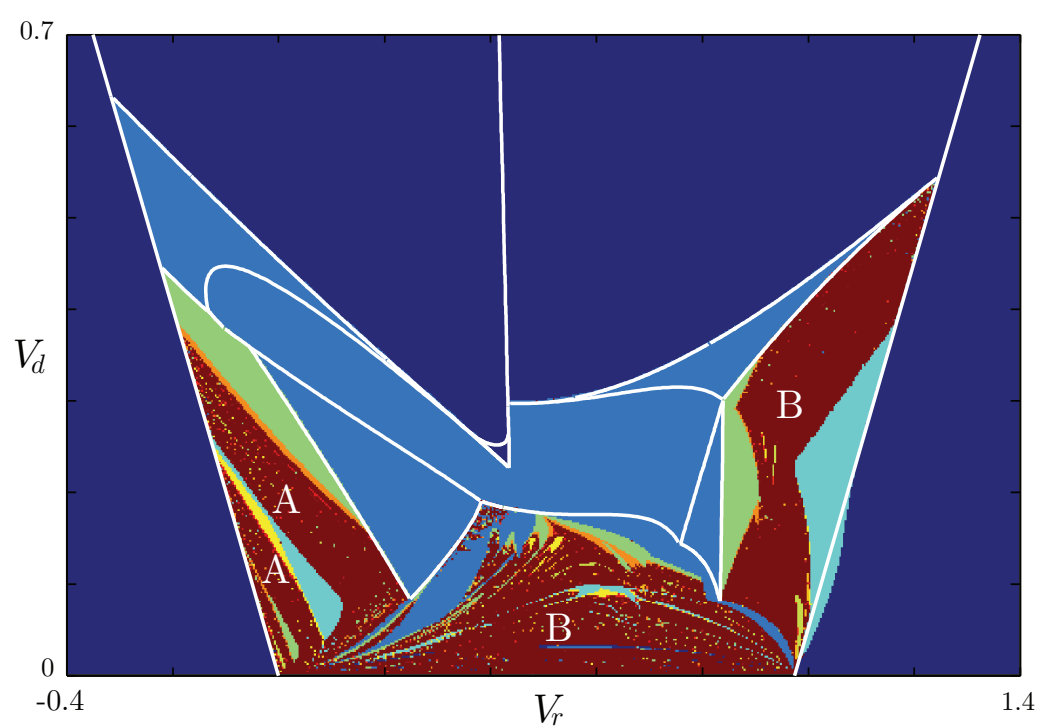

Figure 9. The curves shown in the previous figures are superimposed on a bifurcation diagram obtained through simulation. The colors correspond to limit cycles of different period, going from blue for cycles of period $T$ to red for cycles of period $\geq 16 T$ and for aperiodic attractors. Notice that the stationary solutions on the two sides have period $T$ in the cylindrical state space, even though they correspond to steady states of the electrical variables $v$ and $i$.

chaotic dynamics. To obtain a rough picture of the complex invariant sets found in the rest of the parameter plane, we present some numerical results obtained by direct simulation of the system's equations.

4.2. Numerical simulation. In Figure 9 , we considered a grid of $501 \times 351$ points in the $\left(V_{r}, V_{d}\right)$ parameter plane and simulated the system for $200 T$ with parameter values set at each of the points on the grid. Each point was then colored according to the period of the asymptotic solution exhibited by the system after transient. The resulting two-parameter bifurcation diagram is shown together with the bifurcation curves obtained previously.

Observing the red regions in the diagram, which correspond to chaotic dynamics, we can distinguish regions with two qualitatively different dynamics: one on the left (marked as A), and another one on the bottom and right of the figure (marked as B). A plot of the peakto-peak map (or Lorenz map; see [24]) of the maxima of the current (see Figure 10 for two representative examples) shows that in region A the peak-to-peak map of the attractor is nearly filiform (i.e., points are roughly distributed along one or more curves; see [5]), whereas in region B it is a thick cloud of points. Since the peak-to-peak map is nothing other than a particular Poincaré map, this implies that the chaotic attractor is nearly two-dimensional in region $\mathrm{A}$, while its fractal dimension is between 2 and 3 in region $\mathrm{B}$. In other words, in region A, the behavior of the system at regime is well approximated by that of the one-dimensional map obtained from the peak-to-peak plot, while in region B the chaotic dynamics is more complex.

Copyright (c) by SIAM. Unauthorized reproduction of this article is prohibited. 

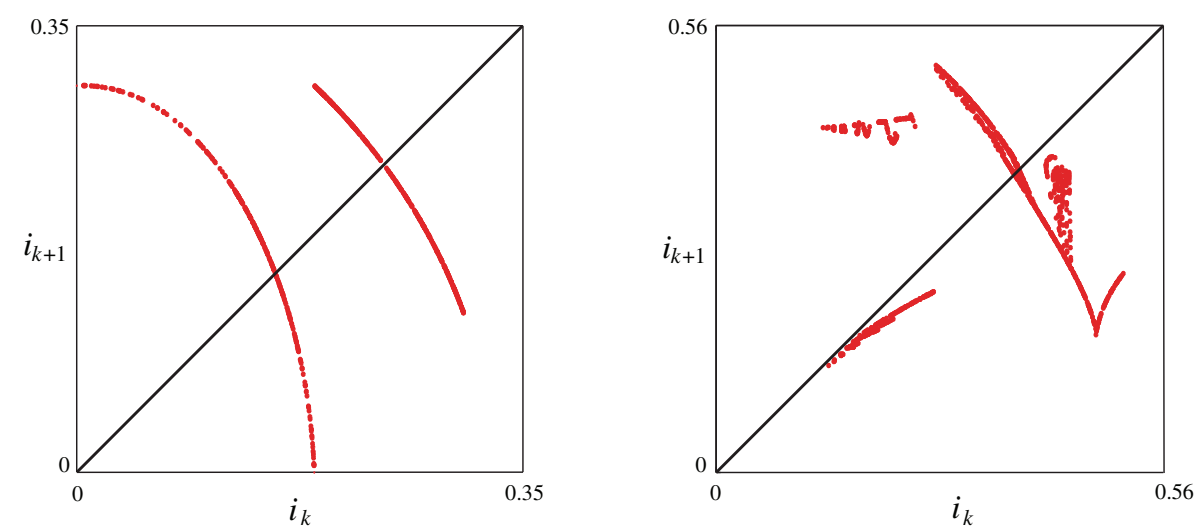

Figure 10. Peak-to-peak plots for $V_{r}=0.1$ and $V_{d}=0.12$ (left panel), and for $V_{r}=0.5$ and $V_{d}=0.1$ (right panel). On the left, the filiform plot indicates that the chaotic attractor has fractal dimension close to 2, which is not the case in the right plot.

5. Bifurcations: Classification. We now illustrate how the DIBs detected numerically can be properly classified analytically, predicting the transitions observed locally to some of them. It is worth mentioning here that, despite the complexity of dynamics shown in the brute-force bifurcation diagram in Figure 9, the main transitions can be clearly accounted for by considering the interaction between smooth and discontinuity-induced bifurcation curves. In particular, we notice the presence of several codimension-two bifurcation points which act as organizing centers for the nearby dynamics. As a telling example, observe the three codimension-two points, $P_{1}, P_{2}$, and $P_{3}$, along the boundary-intersection crossing curve in the center of Figure 8. The importance of these codimension-two points was highlighted in [21], where they were classified as type II codimension-two DIBs, namely as DIBs of nonhyperbolic limit cycles.

Call $N_{a}$ the limit of the linearized Poincaré map for solutions $(1,1,0)$ as they approach the boundary-intersection crossing, and $N_{b}$ the limit of the linearized Poincaré map for solutions $(1,1,1)$ as they approach the boundary-intersection crossing. A method of classification, introduced by $[15,16,17,18]$, allows us to distinguish between different scenarios based on the number of eigenvalues of the matrices $N_{a}, N_{b}, N_{a} N_{a}$, and $N_{a} N_{b}$ that are greater than 1 or smaller than -1 . More precisely, if the total number of eigenvalues of $N_{a}$ and $N_{b}$ greater than 1 is even, a solution $(1,1,0)$ turns into a solution $(1,1,1)$ crossing the bifurcation (persistence scenario). If it is odd, on the other hand, two cycles collide and annihilate at the bifurcation (nonsmooth fold scenario). Then, if the total number of eigenvalues of $N_{a}$ and $N_{b}$ smaller than -1 is odd, a cycle of period $2 T$ is born at the bifurcation (nonsmooth period-doubling scenario); this cycle coexists with the solution $(1,1,0)$ if the total number of eigenvalues of $N_{a} N_{a}$ and $N_{a} N_{b}$ greater than 1 is even, while it coexists with the solution $(1,1,1)$ if this number is odd.

Figure 11 shows the real part of the nontrivial eigenvalues of the matrices $N_{a}, N_{b}, N_{a} N_{a}$, and $N_{a} N_{b}$, evaluated along the boundary-intersection crossing in the center of Figure 8. Notice that, since a periodic orbit comprising a sliding segment is superstable, i.e., has a zero multiplier, matrices $N_{b}$ and $N_{a} N_{b}$ always have an eigenvalue equal to 0 . Additionally, matrices 


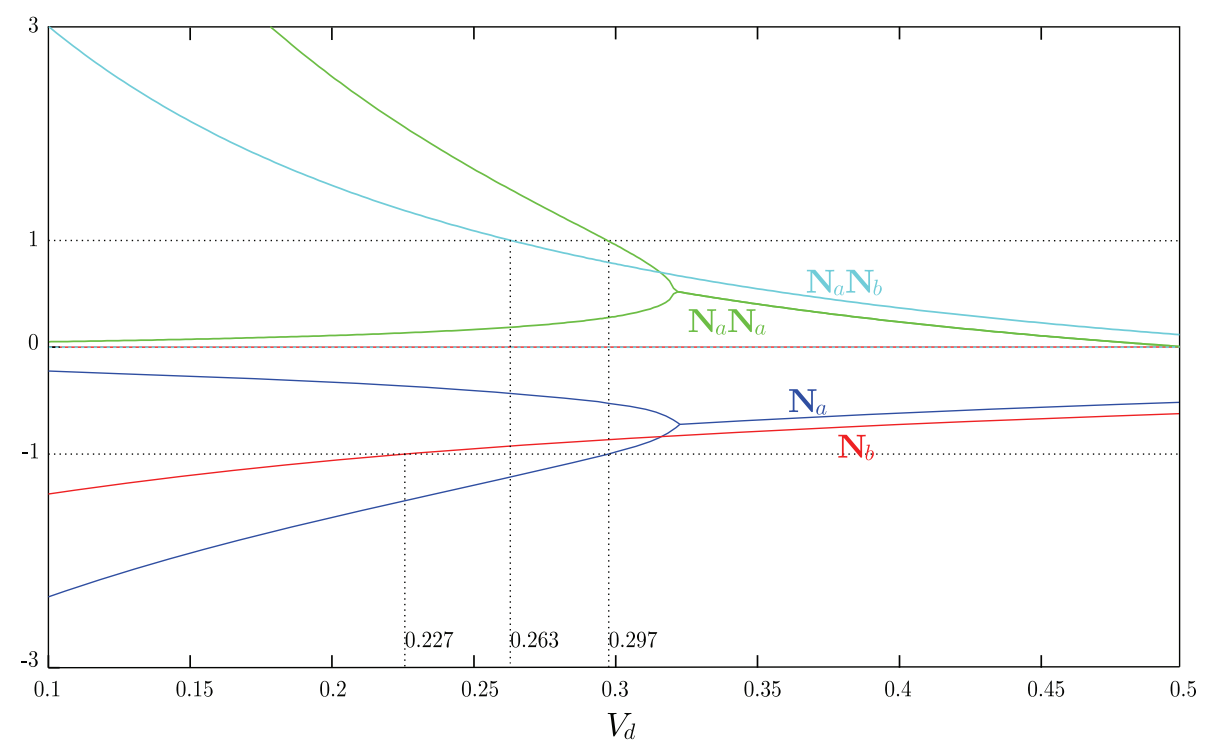

Figure 11. Real parts of the eigenvalues of matrices $N_{a}$ (blue), $N_{b}$ (red), $N_{a} N_{a}$ (green), and $N_{a} N_{b}$ (light blue).

$N_{a}$ and $N_{a} N_{a}$ have complex conjugate eigenvalues for large values of $V_{d}$, where the plots of their real parts coincide in Figure 11. For large values of $V_{d}$, above point $P_{1}$ in Figure 8, the total number of real eigenvalues of $N_{a}$ and $N_{b}$ larger than 1 or smaller than -1 is zero; hence we expect a persistence scenario, where a nonsliding cycle $(1,1,0)$ turns into a sliding cycle $(1,1,1)$. Since all eigenvalues are in the unit circle, the two cycles are stable. At $V_{d}$ equal to 0.297 (point $P_{1}$ ), one of the eigenvalues of $N_{a}$ becomes equal to -1 . Here, the bifurcating cycle is nonhyperbolic so that, as was proved in [8], there must be a flip curve transversally intersecting the boundary-intersection crossing, while another boundary-intersection crossing, tangent to the flip curve, must emanate from the codimension-two point. For $V_{d}$ between 0.297 and 0.263 , one eigenvalue of $N_{a}$ is smaller than -1 , so that we expect a nonsmooth period-doubling scenario. Since the total number of eigenvalues of $N_{a} N_{a}$ and $N_{a} N_{b}$ greater than 1 is odd, the double-period orbit coexists with the $(1,1,1)$ cycle, which is now unstable. At $V_{d}=0.263$ (point $P_{2}$ ), since the matrix $N_{a} N_{b}$, which is the limit of the linearized Poincaré map of the cycle $(2,2,1)$ as it approaches the boundary-intersection crossing, has an eigenvalue equal to 1, we expect a fold bifurcation of the double-period cycle to emanate tangentially to the boundary-intersection crossing (this was again proved in [8]), and for $0.263>V_{d}>0.227$ one eigenvalue of $N_{a} N_{a}$ is larger than 1 , while those of $N_{a} N_{b}$ remain smaller than 1 , so that the double-period cycle born at the boundary-intersection crossing now coexists with the $(1,1,0)$ cycle. At $V_{d}=0.227$ (point $P_{3}$ ), an eigenvalue of $N_{b}$ becomes smaller than -1 . Once again, we expect to find a flip curve transversally intersecting the boundary-intersection crossing. Below this point, both $(1,1,0)$ and $(1,1,1)$ are unstable.

6. Conclusions. We have performed a detailed bifurcation analysis of the buck converter, unveiling an intricate set of dynamics. The simplest periodic regimes have been analyzed, and the boundaries of existence of the periodic attractors have been detected and classified.

Copyright (c) by SIAM. Unauthorized reproduction of this article is prohibited. 
Moreover, the chaotic regions have been explored, through peak-to-peak plots, revealing the existence of different types of chaotic regimes. This study has exploited novel theoretical results and original numerical methods. Further developments in this direction may bring us to a deeper understanding of the dynamics of the converter, and possibly to the exploitation of its complex dynamics in applications.

Appendix. Nondimensionalization of the circuit's equations. A detailed model of the buck converter is provided in Figure 1, where the threshold is assumed to be a negative sawtooth with period $\tilde{T}$, mean value $\tilde{V}_{r}$, and peak-to-peak amplitude $\tilde{V}_{d}$. Calling $\tilde{t}$ the physical time, the equations describing the evolution of the circuit in the variables $\left(v_{c}, i_{l}, \tilde{\theta}\right) \in \mathbb{R}^{2} \times S$ are

$$
\left\{\begin{array}{l}
\frac{d v_{c}}{d \tilde{t}}=-\frac{v_{c}}{R_{c} C}+\frac{i_{l}}{C} \\
\frac{d i_{l}}{d \tilde{t}}=-\frac{v_{c}}{C}-\frac{R_{s}}{L} i_{l}+\frac{V_{i n}}{L} \\
\frac{d \tilde{\theta}}{d \tilde{t}}=1
\end{array}\right.
$$

when the switch is closed;

$$
\left\{\begin{array}{l}
\frac{d v_{c}}{d \tilde{t}}=-\frac{v_{c}}{R_{c} C}+\frac{i_{l}}{C} \\
\frac{d i_{l}}{d \tilde{t}}=-\frac{v_{c}}{C}-\frac{R_{s}}{L} i_{l} \\
\frac{d \tilde{\theta}}{d \tilde{t}}=1
\end{array}\right.
$$

when the switch is open and the diode is directly biased; and

$$
\left\{\begin{array}{l}
\frac{d v_{c}}{d \tilde{t}}=-\frac{v_{c}}{R_{c} C} \\
\frac{d i_{l}}{d \tilde{t}}=0 \\
\frac{d \tilde{\theta}}{d \tilde{t}}=1
\end{array}\right.
$$

when the switch is open and the diode is inversely biased. In order to reduce the number of parameters we rescale the variables as follows:

$$
\begin{gathered}
t=\frac{\tilde{t}}{T_{0}}, \quad \theta=\frac{\tilde{\theta}}{T_{0}}, \quad v=\frac{v_{c}}{V_{i n}}, \quad i=\frac{Z_{0}}{V_{i n}} i_{l}, \quad Q=\frac{R_{c}}{Z_{0}}, \quad Q_{s}=\frac{Z_{0}}{R_{s}}, \\
T=\frac{\tilde{T}}{T_{0}}, \quad V_{r}=\frac{\tilde{V}_{r}}{V_{i n}}, \quad V_{d}=\frac{\tilde{V}_{d}}{V_{i n}}
\end{gathered}
$$


where the characteristic impedance and the natural period of the circuit are $Z_{0}=\sqrt{L / C}$ and $T_{0}=2 \pi \sqrt{L C}$, so that the above equations become

$$
\left\{\begin{array}{l}
\frac{d v}{d t}=-\frac{2 \pi}{Q} v+2 \pi i \\
\frac{d i}{d t}=-2 \pi v-\frac{2 \pi}{Q_{s}} i+2 \pi \\
\frac{d \theta}{d t}=1
\end{array}\right.
$$

when the switch is closed;

$$
\left\{\begin{array}{l}
\frac{d v}{d t}=-\frac{2 \pi}{Q} v+2 \pi i \\
\frac{d i}{d t}=-2 \pi v-\frac{2 \pi}{Q_{s}} i \\
\frac{d \theta}{d t}=1
\end{array}\right.
$$

when the switch is open and the diode is directly biased; and

$$
\left\{\begin{array}{l}
\frac{d v}{d t}=-\frac{2 \pi}{Q} v \\
\frac{d i}{d t}=0 \\
\frac{d \theta}{d t}=1
\end{array}\right.
$$

when the switch is open and the diode is inversely biased.

\section{REFERENCES}

[1] F. Angulo, E. Fossas, And G. Olivar, Transition from periodicity to chaos in a pwm-controlled buck converter with ZAD strategy, Internat. J. Bifur. Chaos Appl. Sci. Engrg., 15 (2005), pp. 3245-3264.

[2] A. E. Aroudi, L. Benadero, E. Toribio, and S. Machiche, Quasiperiodicity and chaos in the DC-DC buck-boost converter, Internat. J. Bifur. Chaos Appl. Sci. Engrg., 10 (2000), pp. 359-371.

[3] L. Benadero, A. E. Aroudi, G. Olivar, E. Toribio, and E. Gomez, Two-dimensional bifurcation diagrams. Background pattern of fundamental DC-DC converters with pwm control, Internat. J. Bifur. Chaos Appl. Sci. Engrg., 13 (2003), pp. 427-451.

[4] W.-J. Beyn, A. Champneys, E. Doedel, W. Govaerts, Yu. A. Kuznetsov, and B. Sandstede, Numerical continuation, and computation of normal forms, in Handbook of Dynamical Systems, 2nd ed., B. Fiedler, ed., Elsevier Science, Burlington, MA, 2002, pp. 149-219.

[5] M. Candaten and S. Rinaldi, Peak-to-peak dynamics: A critical survey, IInternat. J. Bifur. Chaos Appl. Sci. Engrg., 10 (2000), pp. 1805-1819.

[6] K. Chakrabarty, G. Poddar, And S. Banerjee, Bifurcation behavior of the buck converter, IEEE Trans. Power Electronics, 11 (1996), pp. 439-447.

[7] A. Colombo, Boundary intersection crossing bifurcation in the presence of sliding, Phys. D, 237 (2008), pp. 2900-2912.

[8] A. Colombo And F. Dercole, Discontinuity induced bifurcations of nonhyperbolic cycles in nonsmooth systems, SIAM J. Appl. Dyn. Syst., submitted.

Copyright (C) by SIAM. Unauthorized reproduction of this article is prohibited. 
[9] F. Dercole And Yu. A. Kuznetsov, SlideCont: An Auto 97 driver for bifurcation analysis of Filippov systems, ACM Trans. Math. Software, 31 (2005), pp. 95-119.

[10] M. Di Bernardo, C. J. Budd, And A. R. Champneys, Normal form maps for grazing bifurcations in n-dimensional piecewise-smooth dynamical systems, Phys. D, 160 (2001), pp. 222-254.

[11] M. di Bernardo, C. J. Budd, A. R. Champneys, and P. Kowalczyk, Piecewise-Smooth Dynamical Systems: Theory and Applications, Springer-Verlag, Berlin, 2008.

[12] M. di Bernardo, E. Fossas, G. Olivar, And F. Vasca, Secondary bifurcation and high periodic orbits in voltage controlled buck converter, Internat. J. Bifur. Chaos Appl. Sci. Engrg., 7 (1997), pp. 27552771.

[13] E. J. Doedel, H. B. Keller, And J.-P. Kernévez, Numerical analysis and control of bifurcation problems: (I) Bifurcation in finite dimensions, Internat. J. Bifur. Chaos Appl. Sci. Engrg., 1 (1991), pp. $493-520$.

[14] E. J. Doedel, H. B. Keller, And J.-P. Kernévez, Numerical analysis and control of bifurcation problems: (II) Bifurcation in infinite dimensions, Internat. J. Bifur. Chaos Appl. Sci. Engrg., 1 (1991), pp. $745-772$.

[15] M. I. FEIGIN, Doubling of the oscillation period with C-bifurcations in piecewise continuous systems, PMM J. Appl. Math. Mech., 34 (1970), pp. 861-869.

[16] M. I. FEIGIN, On the generation of sets of subharmonic modes in a piecewise continuous system, PMM J. Appl. Math. Mech., 38 (1974), pp. 810-818.

[17] M. I. FeIGin, On the structure of C-bifurcation boundaries of piecewise continuous systems, PMM J. Appl. Math. Mech., 42 (1978), pp. 820-829.

[18] M. I. Feigin, Forced Oscillations in Systems with Discontinuous Nonlinearities, Nauka, Moscow, 1994 (in Russian).

[19] A. F. Filippov, Differential Equations with Discontinuous Righthand Sides, Kluwer Academic Publishers, Dordrecht, The Netherlands, 1988.

[20] J. G. Kassakian, M. F. Schlecht, and G. C. Verghese, Principles of Power Electronics, AddisonWesley, Reading, MA, 1991.

[21] P. Kowalczyk, M. di Bernardo, A. R. Champneys, S. J. Hogan, M. Homer, P. T. Piironinen, Yu. A. Kuznetsov, And A. Nordmark, Two-parameter discontinuity-induced bifurcations of limit cycles: Classification and open problems, Internat. J. Bifur. Chaos Appl. Sci. Engrg., 16 (2006), pp. 601-629.

[22] S. Maity, D. Tripathy, T. K. Bhattacharya, and S. BanerJee, Bifurcation analysis of PWM-1 voltage-mode-controlled buck converter using the exact discrete model, IEEE Trans. Circuits Systems I Fund. Theory Appl., 54 (2007), pp. 1120-1130.

[23] N. Mohan, T. M. Undeland, and W. P. Robbins, Power Electronics: Converters, Applications, and Design, John Wiley \& Sons, New York, 2003.

[24] S. H. Strogatz, Nonlinear Dynamics and Chaos, Addison-Wesley, Reading, MA, 1994.

[25] P. Thota And H. Dankowicz, TC-HAT $\widehat{(T C)}$ : A novel toolbox for the continuation of periodic trajectories in hybrid dynamical systems, SIAM J. Appl. Dyn. Syst., 7 (2008), pp. 1283-1322.

Copyright (C) by SIAM. Unauthorized reproduction of this article is prohibited. 